\title{
Compact optical fiber sensor smart node
}

Seth W. Lloyd

seth.lloyd@stanford.edu

Jason A. Newman

Daniel R. Wilding

Richard H. Selfridge

selfridge@ee.byu.edu

Stephen M. Schultz

Follow this and additional works at: https://scholarsarchive.byu.edu/facpub

Part of the Electrical and Computer Engineering Commons

\section{Original Publication Citation}

Lloyd, Seth W., Jason A. Newman, Daniel R. Wilding, Richard H. Selfridge, and Stephen M.

Schultz. "Compact optical fiber sensor smart node." Review of Scientific Instruments 78 (27)

\section{BYU ScholarsArchive Citation}

Lloyd, Seth W.; Newman, Jason A.; Wilding, Daniel R.; Selfridge, Richard H.; and Schultz, Stephen M., "Compact optical fiber sensor smart node" (2007). Faculty Publications. 267.

https://scholarsarchive.byu.edu/facpub/267 accepted for inclusion in Faculty Publications by an authorized administrator of BYU ScholarsArchive. For more information, please contact ellen_amatangelo@byu.edu. 


\title{
Compact optical fiber sensor smart node
}

\author{
Seth W. Lloyd, Jason A. Newman, Daniel R. Wilding, \\ Richard H. Selfridge, and Stephen M. Schultz \\ Department of Electrical and Computer Engineering, Brigham Young University, 464 CB, Provo, Utah 84602
}

(Received 26 September 2006; accepted 20 February 2007; published online 22 March 2007)

\begin{abstract}
We present a new optical fiber sensor interrogator specifically designed for an embedded instrumentation system. The proposed system consists of a super luminescent diode as a broadband source, a high speed tunable micro-electro-mechanical system (MEMS) filter, photodetector, and an integrated microprocessor for data aggregation, processing, and communication. The entire system is integrated together in a compact package to create a fiber "smart" sensor. The system is capable of interrogating a variety of multiplexed fiber sensors, processing the data, and communicating the results digitally. As an example, the system has been calibrated with an array of fiber Bragg grating sensors. () 2007 American Institute of Physics. [DOI: 10.1063/1.2715994]
\end{abstract}

\section{INTRODUCTION}

Optical fiber sensors enable nonintrusive system evaluation because of their small size, flexibility, and durability, while also exhibiting immunity to electromagnetic interference and resistance to corrosive materials. They are finding significant use in health monitoring and analysis of civil, ${ }^{1}$ mechanical, and aerospace systems. ${ }^{2}$ As the applications for optical sensors grow, so does the need for optical sensor monitoring systems.

While traditional sensor interrogation requires the measurement of an electrical phenomenon such as potential or resistance, fiber sensor interrogators must measure optical phenomena. For example, Fig. 1 shows the transmission and reflection spectrum of an array of fiber Bragg grating (FBG) sensors. Each individual FBG sensor reflects a narrow band of wavelengths at the Bragg wavelength, allowing the remaining optical power to be transmitted. The center wavelength of this reflected band, or peak, shifts in the presence of different environmental phenomena, such as temperature or strain. Therefore, sensor interrogation requires the measurement and analysis of the spectral features in the wavelength domain. Similar wavelength-based sensor interrogation is required for other optical sensors, such as Fabry-Pérot and long period grating sensors.

Many groups have begun to assess the need for optical sensor interrogators through a variety of demodulating schemes. Traditionally, demodulation schemes employ one of two different methods. One approach uses a diffraction grating to demultiplex an optical signal into narrow wavelength bands which can then be measured by a photodetector array. ${ }^{3,4}$ Such systems facilitate high speed interrogation because multiple channels can be acquired simultaneously. However, increasing the available spectral bandwidth requires the use of larger and larger photodetector arrays. Such systems are also generally unable to demultiplex densely spaced channels.

Another approach uses a scanning Fabry-Pérot interferometer to separate an optical signal into single wavelength channels one at a time. ${ }^{5,6}$ Because each channel is scanned separately, this method requires only a single photodetector to measure all channels. Additionally, a high finesse filter allows for monitoring of densely spaced channels. However, tuning speeds for Fabry-Pérot interferometers are generally low, greatly limiting the possible interrogation speed.

Even with current advances, many optical sensor monitoring systems are large and bulky, costly, have high power consumption, and perform only the most basic functions. Thus, while the sensor itself is extremely compact and adaptable to a variety of situations, the interrogation system is not. The large size limits the possibilities for sensor applications in embedded instrumentation systems. Additionally, such systems are often stand-alone units and lack the capability of integration into existing instrumentation systems.

We present an alternative fiber sensor interrogation system designed specifically for embedded systems applications. As such, our fiber sensor integrated monitor (FSIM) incorporates all of the components necessary for interrogating, processing, and communicating data collected from multiplexed fiber sensors into a compact package creating, when combined with a fiber sensor, a "smart" sensor node.

The FSIM integrates all of the components necessary for measuring and analyzing wavelength based data onto a single circuit board. The FSIM also exhibits a dramatic decrease in power as well as an increase in operating speed. However, the most significant advantage of such a system is the ability to act as a "smart" sensor. Smart sensors enhance traditional sensor functionality by adding signal conditioning, data processing, and a bus output at the sensor node. ${ }^{7}$ Smart sensors have added functionality over traditional sensors and can be networked together to create powerful distributed sensor systems. Additionally, smart sensors can receive and send commands, giving the smart sensor the ability to make decisions based upon the data and commands received. It is the smart sensor capabilities of the FSIM that enables the creation of new embedded sensor applications for fiber sensors. 


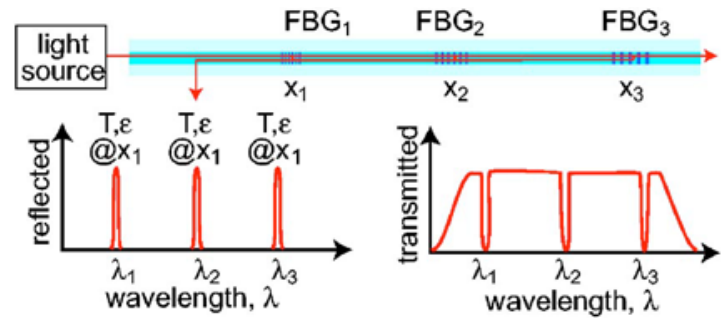

FIG. 1. Three-element fiber Bragg grating array in reflection and transm ission.

\section{SMART SENSOR OVERVIEW}

Figure 2 shows a block diagram of the operation of the FSIM platform. In this smart sensor architecture the controller is programmed to properly decode the incoming data, control the optical source according to measurement needs, read and interpret the data from the detector, and then format the data and return it to the data bus. Some advantages of this system are its ability to adapt to a wide variety of data formats and make the required measurements. In addition, the FSIM platform is capable of being configured for the interrogation of a wide range of sensor types such as fiber Bragg grating sensors, long period grating sensors, Fabry-Pérot sensors, etc.

The integration of source, detector, and controller creates an adaptable smart sensor with a wide range of possible applications and uses in a compact package. In our FBG sensor example, we developed algorithms for the controller that perform several high level tasks such as

- Scan the entire spectrum: The controller incrementally scans the filter across its operating range. At each step the optical power is acquired and stored for analysis.

- Find and track peaks: Software has been developed to scan the entire spectrum and detect individual peaks. The initial scan is performed at a low resolution. Once peaks are identified, the controller increases the scan resolution and zooms in on the individual peaks, tracking any changes in the peak wavelength. The simple peak tracking algorithm results in a dramatic increase in tracking speed by reducing the size of the scan. The peak tracking algorithm has been designed to track multiple peaks.

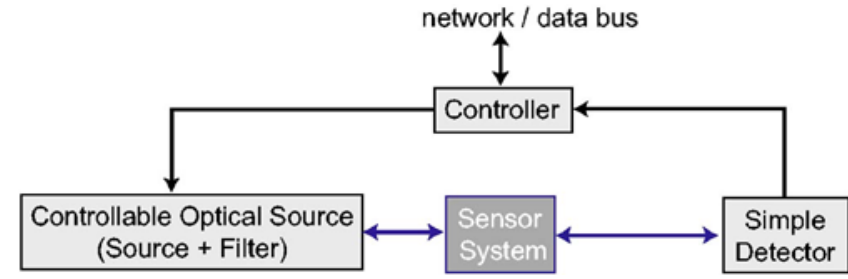

FIG. 2. Block diagram of FSIM system operation.

- Self calibration: Using a method developed specifically for the FSIM, the controller utilizes an integrated reference to calibrate the wavelength accuracy of the system on each scan.

These are a few examples of the features that have been developed for the FSIM platform. However, the integrated controller allows for considerable expansion. For example, software could be written allowing a user to instruct the FSIM to sample a sensor response once every $5 \mathrm{~min}$, compare the result with previous results, and notify the user only if there is a significant change in the measurand. Such adaptations are essential for allowing large distributed networks of sensors since they free up the central processing unit by performing much of the data processing and analysis at the sensor node.

\section{INSTRUMENT OPERATION}

The FSIM operates by scanning across a wavelength band, recording the spectral response of the sensor, and calibrating the results to a fixed wavelength reference.

\section{A. Scanning}

The controllable optical source is created by the combination of a fixed optical source with broad wavelength content and a tunable optical filter with a narrow transmission band. Figure 3 shows how these components combine to produce a narrowband tunable optical source that can be used to sample the spectral response of the fiber sensor. The broadband source $P_{\text {source }}(\lambda)$ is transmitted through the filter $T_{\text {filter }}\left(\lambda_{V}\right)$, where the center wavelength $\lambda_{V}$ of the filter transmission depends on the applied voltage $V$. The filtered output

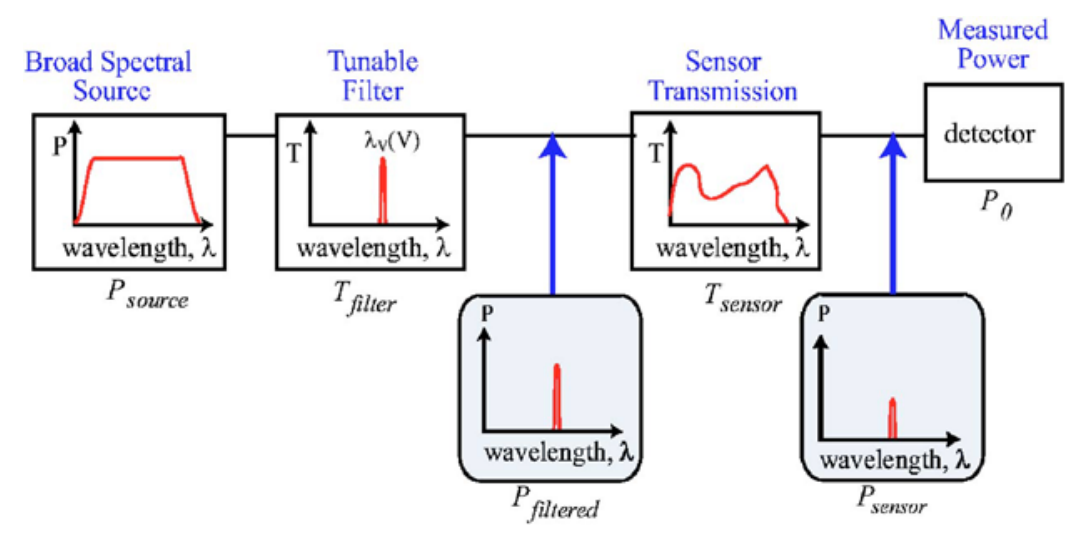

FIG. 3. FSIM controllable sampling of spectral location. 


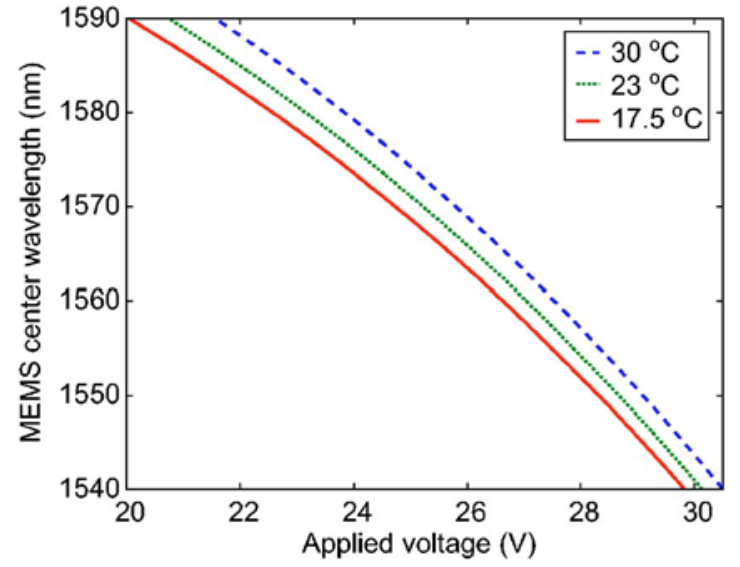

FIG. 4. Temperature drift of the MEMS filter response.

$$
P_{\text {filtered }}(\lambda)=P_{\text {source }}(\lambda) \cdot T_{\text {filter }}\left(\lambda_{V}\right)
$$

is simply the product of the optical source $P_{\text {source }}$ and the filter response $T_{\text {filter }}$. The resulting filtered optical source $P_{\text {filtered }}(\lambda)$ is an impulse function whose center wavelength depends on the applied voltage $V$. The filtered source $P_{\text {filtered }}$ is transmitted through a fiber sensor with spectral response $T_{\text {sensor }}(\lambda)$. The sampled response

$$
P_{\text {sensor }}(\lambda)=T_{\text {sensor }}(\lambda) \cdot P_{\text {filtered }}(\lambda)
$$

is measured with a simple optical detector resulting in

$$
\begin{aligned}
& P_{0}(V)=\int P_{\text {sensor }}(\lambda) d \lambda, \\
& \propto T_{\text {sensor }}\left(\lambda_{V}\right) .
\end{aligned}
$$

The FSIM can read the entire spectral response of the sensor by incrementally adjusting the applied voltage $V$ to scan across the spectrum. The spectral response is read by serially sampling the spectrum in $n$ discrete steps. The result is a recreation of the spectral response $T_{\text {sensor }}$ that is a function of the applied voltage $V$. The voltage $V$ is then converted into the corresponding wavelength resulting in the measured response as a function of $\lambda$.

\section{B. Wavelength calibration}

Figure 4 shows that the filter response is nonlinear and depends on the temperature of the filter. The temperature dependence can be offset by using the thermoelectric cooler (TEC) integrated into the filter package. However, to avoid the high power consumption caused by using a TEC, and because the FSIM already has an integrated microcontroller, an algorithm was developed to compensate for the temperature drift of the filter.

In order to determine the relationship between the voltage and wavelength, the FSIM is connected to a thermal compensated grating (TCG). A TCG is packaged in such a way that there is an insignificant shift in the Bragg wavelength with temperature. ${ }^{10}$ By connecting the FSIM to a TCG, it receives a specific wavelength reference each scan. The calibration algorithm uses the voltage corresponding to the known wavelength reference to adjust the voltagewavelength relationship.

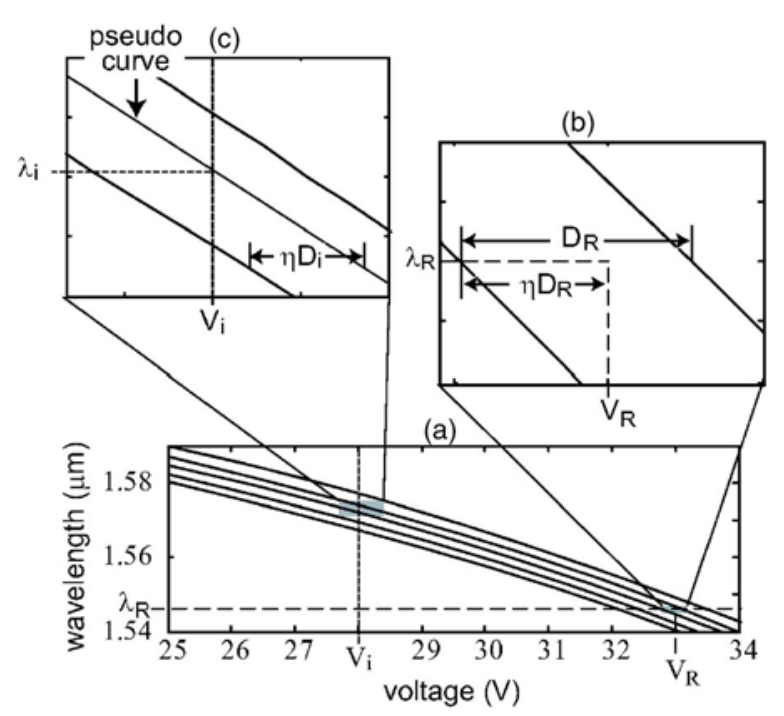

FIG. 5. Graphical representation of the FSIM calibration process.

To characterize the response, a voltage versus wavelength curve, such as those in Fig. 4, is measured for various filter temperatures. At each filter temperature we make sure that the measured data includes the wavelength of the TCG, $\lambda_{R}$. The measured voltage $V_{R}$ corresponding to the known reference is then compared against the measured data. Figure 5(a) shows representative voltage versus wavelength curves. The actual number of measured curves is larger than what is shown in Fig. 5. Since the measured reference voltage $V_{R}$ will most likely lie between curves, this voltage is used to determine the relative distance between curves $\eta$. Figure 5(b) shows the calculation of this relative distance $\eta$. Once the relative distance $\eta$ is known, a pseudocurve is generated, which has a relative separation of $\eta$ between the two closest curves. The wavelength corresponding to an arbitrary voltage $V_{i}$ is the intersection of this pseudocurve and the voltage of interest $V_{i}$. Every time that the spectrum is scanned the wavelength calibration is performed.

\section{INSTRUMENT DESCRIPTION}

Figure 6 shows the primary components of the FSIM. They consist of a superluminescent diode (SLD) as a broadband source, a high-speed tunable micro-electro-mechanical system (MEMS) filter, a pin photodiode, and a microprocessor for data processing, aggregation, and transmission. By integrating a microcontroller into the sensor system, the FSIM effectively becomes a smart sensor. The source, filter, and detector can all be controlled and monitored by the microprocessor. Data can be collected and processed by the microcontroller before being transmitted onto the data bus.

\section{A. Superluminescent diode}

The optical source needs to meet the following requirements (1) small size, (2) low power consumption, (3) broad wavelength content, and (4) efficient fiber coupling. To meet these requirements we used the EXS1505 superluminescent diode from Exalos. This device has both good fiber coupling efficiency and a wide spectral content (about $30 \mathrm{~nm}$ ). It is 


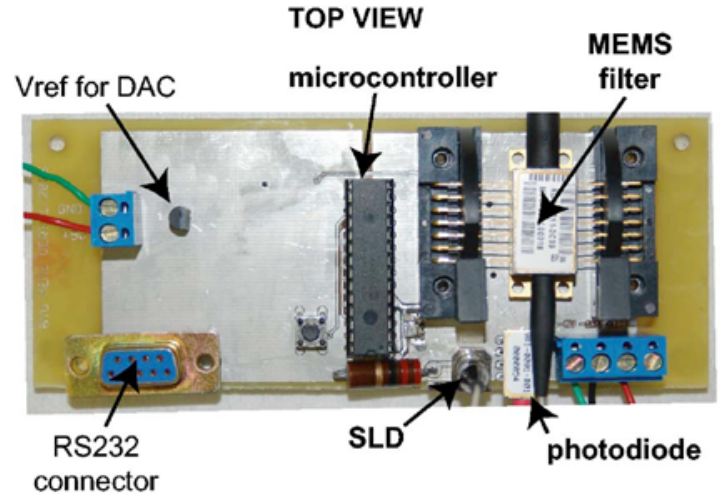

connector

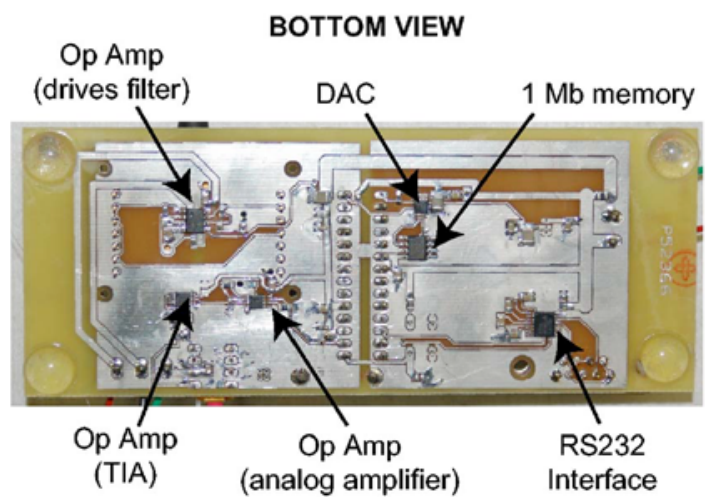

FIG. 6. FSIM board.

also small in size and has low power consumption. In order to conserve power, no active cooling is applied to the SLD.

\section{B. Tunable optical filter}

The MEMS filter used in the FSIM is the MT-15 Tunable Filter made by Nortel Networks. It consists of two thin film membranes separated by a small air gap. These two reflective membranes create a Fabry-Pérot cavity. The FabryPérot cavity transmits a narrow wavelength band and reflects all other wavelengths. The specific wavelength that is transmitted depends on the spacing of the membranes. The membrane separation, and thus the transmitted wavelength, is varied by applying a voltage in the range of 0-40 V. The filter has a nominal tuning speed of $10 \mathrm{~nm} / \mu$ s with a free spectral range greater than $150 \mathrm{~nm}^{8}$ The fast tuning speed of the device is a dramatic improvement over previous speed limitations for Fabry-Pérot based interrogators.

Figure 7 shows the measured transmission for an applied voltage of $15 \mathrm{~V}$. This measurement shows that the full width at half maximum (FWHM) is approximately $60 \mathrm{pm}$. The width of this transmission peak is very consistent over the entire tuning range of the filter.

\section{Electronics}

The smart sensor features of the FSIM are possible because it is controlled by its own onboard microcontroller, the PIC18F2680 from Microchip. The microcontroller performs several key functions in managing the overall FSIM operation. The 12 microcontroller uses a digital to analog converter (DAC) together with external amplifiers to establish the tuning voltage applied to the MEMS filter. An InGaAs

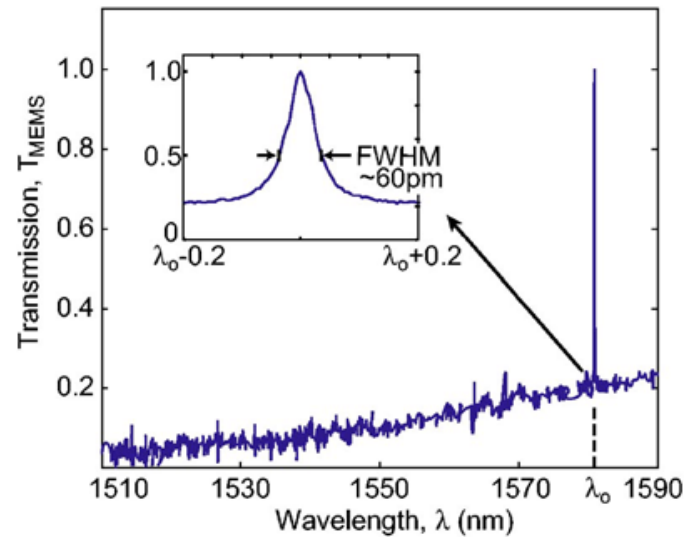

FIG. 7. Spectral linewidth of the transmission band of the MEMS filter.

pin photodiode and transimpedance amplifier convert the received optical power $P_{o}$ (see Fig. 3) into an electrical signal. The electrical signal is acquired by an integrated analog to digital converter (ADC) located on the microcontroller. The microcontroller then processes the data extracting information such as the number of spectral peaks, peak locations, etc. This information can be stored locally on the microcontroller's internal memory, stored externally on an additional on-board memory unit, or transmitted to an external CPU via the data bus. In our current setup, data is transmitted to a desktop computer using the RS232 interface. However, because the sensor data is already in a convenient digital format, data transfer could be accomplished by a variety of means, including WiFi, Bluetooth, Ethernet, etc.

Using the microcontroller and supporting electronics, application specific software can be developed, creating a highly adaptable, tightly controlled smart sensor. For example, rather than scanning the entire spectral response of the sensor, the sampled spectrum can be centered in on specific features such as sensor peaks, ignoring unused wavelength bands. The operation of the FSIM can also be controlled by commands sent from a network or data bus, allowing multiple sensor nodes to communicate with each other or with a central processing unit.

\section{INSTRUMENT CHARACTERIZATION}

In order to characterize the FSIM, a four FBG array fabricated by O-E Land, Inc. was installed in series with a single thermally compensated grating (TCG). Each grating in the FBG array had a nominal FWHM of $0.2 \mathrm{~nm}$ with center wavelengths spaced approximately $4 \mathrm{~nm}$ apart beginning at $1552 \mathrm{~nm}$. All gratings had a reflectivity greater than $99 \%$. The TCG also had a FWHM of $0.2 \mathrm{~nm}$ and a center wavelength of $\sim 1546 \mathrm{~nm}$. Figure 8 shows how the gratings were monitored by the FSIM in reflection using a fiber coupler.

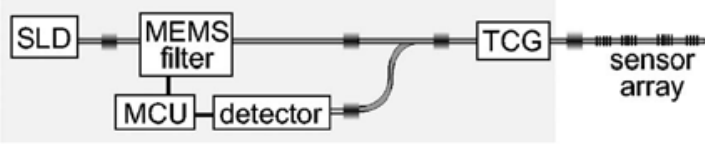

FIG. 8. FSIM test setup using splitter. 


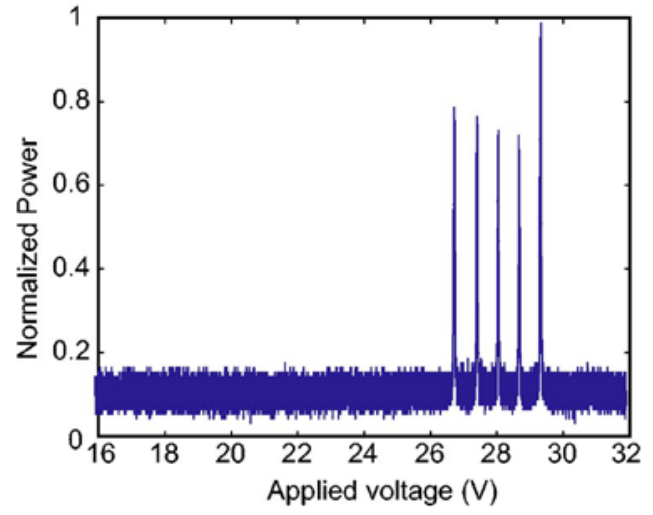

FIG. 9. Spectrum obtained by FSIM of four-grating array with reference grating.

Figure 9 shows the full spectrum obtained by the FSIM. This plot gives the optical power as a function of the applied voltage at the filter. Using the TCG as the reference point $V_{R}$ the FSIM calculates the spectral location of each peak in terms of wavelength. This calculation could similarly be performed for each data point in the scan. However, since demodulation of a fiber sensor typically involves only the spectral location of the peak center wavelength, data processing complexity and scan time are reduced by only performing the calculation for the peak locations. Figure 10 shows the spectrum obtained by the FSIM after locating the peaks. As illustrated, the FSIM scans a small window around each peak and then "jumps" to the next peak. The jumping reduces the number of data points that the FSIM is required to collect, thus; speeding up the collection process. The windowing feature is possible because of the FSIM smart sensor architecture.

The MEMS filter used in the FSIM has a transmission spectrum with a FWHM of 60 pm (see Fig. 7). Thus, the FSIM does not distinguish between spectral features in a sensor that are closer than $60 \mathrm{pm}$. In other words, the spectrum measured by the FSIM is essentially smoothed eliminating very fine spectral features. However, while such distortion is generally undesirable, for many fiber sensor applications it is insignificant. For FBG sensors the reflection peaks, which are coarse spectral features, are more important than the fine spectral features.

In order to demonstrate the spectral smoothing, a surface

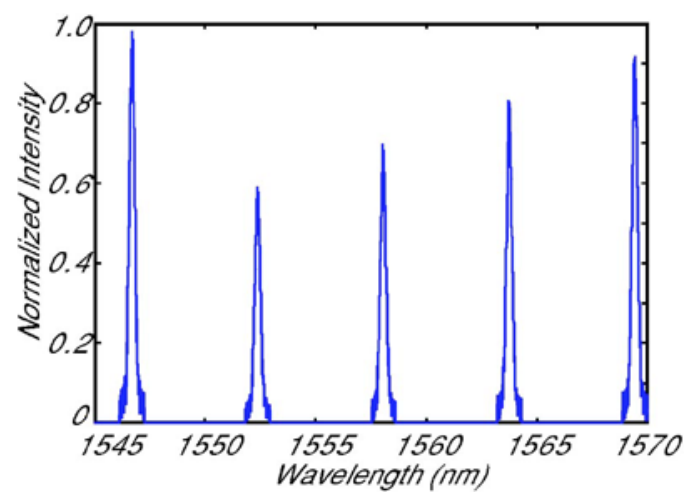

FIG. 10. Spectrum zoomed in on individual peaks.

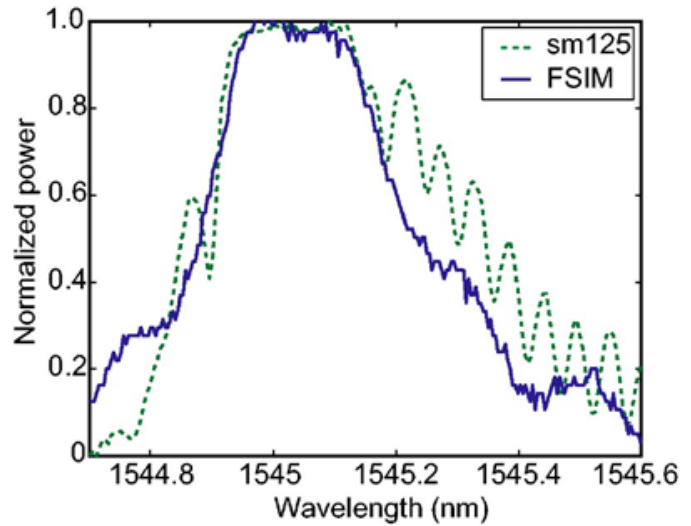

FIG. 11. Resolution comparison of FSIM and Micron Optics sm125 fiber sensor interrogator.

relief fiber Bragg grating was used because of its fine spectral features. ${ }^{9}$ The spectral response of the surface relief FBG was measured using both the FSIM and a sm125 Optical Sensing Interrogator from Micron Optics, which is a high resolution and high precision system designed specifically for interrogating fiber Bragg grating sensors. Figure 11 shows that both measurement techniques produce approximately the same peak location even though the FSIM has smoothed out the fine spectral features. To measure the relative precision of the FSIM at measuring the center of reflection peaks, a grating was heated in small increments and the shifts in peak location were measured using both the FSIM and the sm125. The results showed that the FSIM measured changes in peak wavelength down to about $10 \mathrm{pm}$ For a standard FBG with a temperature response of $\sim 16 \mathrm{pm} /{ }^{\circ} \mathrm{C}$, the temperature precision is approximately $0.6{ }^{\circ} \mathrm{C}$.

\section{CONCLUSION}

We have presented a novel fiber sensor integrated monitor (FSIM) for embedded systems applications. Table I summarizes some of the key specifications of the FSIM. In addition to its compact size and low power consumption, the FSIM also functions as a smart sensor node in which the interrogator performs data analysis, aggregation, formatting, and communications. By adding smart sensor capabilities to sensor monitoring systems, new sensor systems will be possible with increased functionality and adaptability. Furthermore, distributed sensing systems integrating fiber sensors with other sensor technologies will be possible.

TABLE I. Summary of the FSIM specifications.

\begin{tabular}{ll}
\hline \hline \multicolumn{1}{c}{ Parameter } & FSIM capability \\
\hline Bandwidth & $30 \mathrm{~nm}$ \\
Resolution & $0.06 \mathrm{~nm}$ \\
Relative accuracy & $\sim 0.01 \mathrm{~nm}$ \\
Power consumption & $1.5 \mathrm{~W}$ \\
Weight (no power supply) & $102 \mathrm{~g}$ \\
Board dimensions & $6 \mathrm{~cm} \times 19 \mathrm{~cm}$ \\
\hline \hline
\end{tabular}




\section{ACKNOWLEDGMENTS}

The authors would like to thank the Test Resource Management Center (TRMC) Test and Evaluation/Science and Technology (T\&E/S\&T) Program for their support. This work is partially funded by the T\&E/S\&T Program through the Naval Sea Systems Command Warfare Center, Newport, Rhode Island, Contract No. N66604-05-C-2201.

${ }^{1}$ J. Lopez-Higuera, Handbook of Optical Fibre Sensing Technology (Wiley, New York, 2002).

${ }^{2}$ E. Udd, W. Schulz, J. Seims, and M. Morell, Proc. SPIE 3674, 139 (1999).
${ }^{3}$ A. A. Chtcherbakov and P. L. Swart, J. Lightwave Technol. 22, 1543 (2004).

${ }^{4}$ Y. Sano and T. Yoshino, J. Lightwave Technol. 21, 132 (2003).

${ }^{5}$ L. West, D. J. Eigen, and A. Chavez-Pirson, Proc. SPIE 5278, 56 (2003).

${ }^{6}$ A. Unamuno, L. Li, and D. Uttamchandani, IEEE J. Sel. Top. Quantum Electron. 10, 358 (2004).

${ }^{7}$ E. Jacobsen, in Northcon/96: IEEE Technical Applications Conference (Electrical and Electronics Engineers, Piscataway, NJ, 1996), pp. 285290.

${ }^{8}$ Nortel Networks, Preliminary Datasheet for MT-15 Tunable Filter, 2002.

${ }^{9}$ K. H. Smith, B. L. Ipson, T. L. Lowder, A. R. Hawkins, R. H. Selfridge, and S. M. Schultz, Proc. SPIE 5758, 28 (2005).

${ }^{10}$ Yu L. Lo and C. P. Kuo, IEEE Trans. Adv. Packag. 25, 50 (2002). 